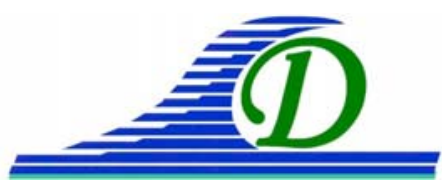
XIII İmes Journées Nationales Génie Côtier - Génie Civil
Dunkerque, 2-4 juillet 2014

DOI:10.5150/jngcgc.2014.093 @ Editions Paralia CFL

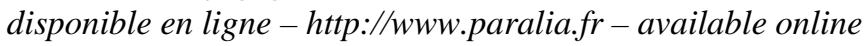

\title{
Le littoral de Cap de l'Eau entre fragilité du milieu physique et impact des pressions anthropiques (Maroc nord-est)
}

\author{
Abdelouahid SAIDI ${ }^{1}$, Abdelkader SBAI ${ }^{1}$, Hafid BOULHOUAL $^{1}$ \\ 1. Université Mohammed Ier, Département de Géographie, Oujda, Maroc. \\ cap_saidi@hotmail.com
}

\section{Résumé :}

Le littoral de Cap de l'Eau est situé au nord-est du Maroc sur la façade méditerranéenne. Il abrite à la fois des côtes rocheuses et des plages sableuses et connait d'importants problèmes environnementaux. Ces problèmes d'origine naturelle et anthropique sont autant des contraintes pour le milieu que pour les ressources naturelles. Les changements survenus au niveau des profils du trait de côte, les variations granulométriques des compartiments de la plage, l'augmentation de la salinité dans les sols et les eaux de l'aquifère phréatique furent déjà des indices de dégradation et la signature d'une sensibilité de la zone côtière envers l'érosion marine. La frange côtière de Cap de l'Eau est sollicitée malgré ces éléments de fragilisation pour différents aménagements : tourisme, urbanisation, infrastructure de base (port, rocade méditerranéenne, ...). La présente note a pour objectif d’aborder la dynamique actuelle de l'érosion côtière du littoral de Cap de l'Eau dans son contexte anthropique, en relation avec les risques climatiques, et d'évaluer les répercussions des formes d'aménagements sur les états physiques des ressources du milieu. La méthodologie adoptée s'appuie sur une analyse diachronique de photographies aériennes et d'images satellitales sur plusieurs années couplées à l'étude granulométriques des sables de la plage. La visualisation et la spatialisation des problèmes sont illustrées grâce aux outils de SIG et de la télédétection.

Mots-clés : Trait de côte, Erosion côtière, Aménagement, Cap de l’Eau, Maroc nord-est.

\section{Introduction}

Les pressions multiples qu'exerce l'occupation humaine constituent un fait généralisé sur les littoraux du Maroc. Le littoral de Cap de l'Eau (nord-est du Maroc) n’échappe pas à cette règle. Considéré longtemps sous occupé et vierge, il est exposé actuellement à une surexploitation de ses ressources naturelles conjuguée à une absence d'une législation dissuasive et une sensibilisation efficace de la population. Ceci empêche la mise en place d'une gestion rationnelle et intégrée des ressources de l'espace littoral.

\section{Présentation de la zone d'étude}

Le littoral de Cap de l’Eau s’ouvre largement sur la mer Méditerranéenne (figure 1). Il est bordé au sud par le massif montagneux de Kebdana et s'étend à l'est jusqu'à Oued 


\section{Thème 6 - Gestion durable des zones littorales et estuariennes}

Moulouya. Ce littoral possède des potentialités et une richesse paysagère qui reste fragile.

La plage de Cap de l'Eau est très réputée par son étendue et sa qualité, aussi belle au niveau de la finesse du sable que pour la qualité des eaux de baignade. Elle s'étend sur une bande de $7 \mathrm{~km}$ avec une largeur variable.

L'embouchure de la Moulouya est un Site d'Intérêt Biologique et Ecologique (SIBE) classé selon la convention du Ramsar (2005) riche en biodiversité animale et végétale.

Sur le plan géologique, le littoral de Cap de l'Eau fait partie du piedmont nord du massif de Kebdana ayant subi une vaste dépression synclinale ondulée par les mouvements néotectoniques et remplie par les sédiments d'aire néogène et quaternaire. Il est marqué par une prépondérance des formations meubles (sable, limon, calcarénite) favorable à l'érosion marine et éolienne.

Sur le plan géomorphologique, les falaises vives du littoral de Cap de l'Eau subissent des phénomènes d'érosion liés au mouvement du plan d'eau d'une part et, d'autre part des phénomènes chimiques et biologiques liés à l'agressivité de l'eau saline.

Le climat de la zone des Kebdana est de type méditerranéen. Le régime pluviométrique est caractérisé par une irrégularité interannuelle des précipitations.

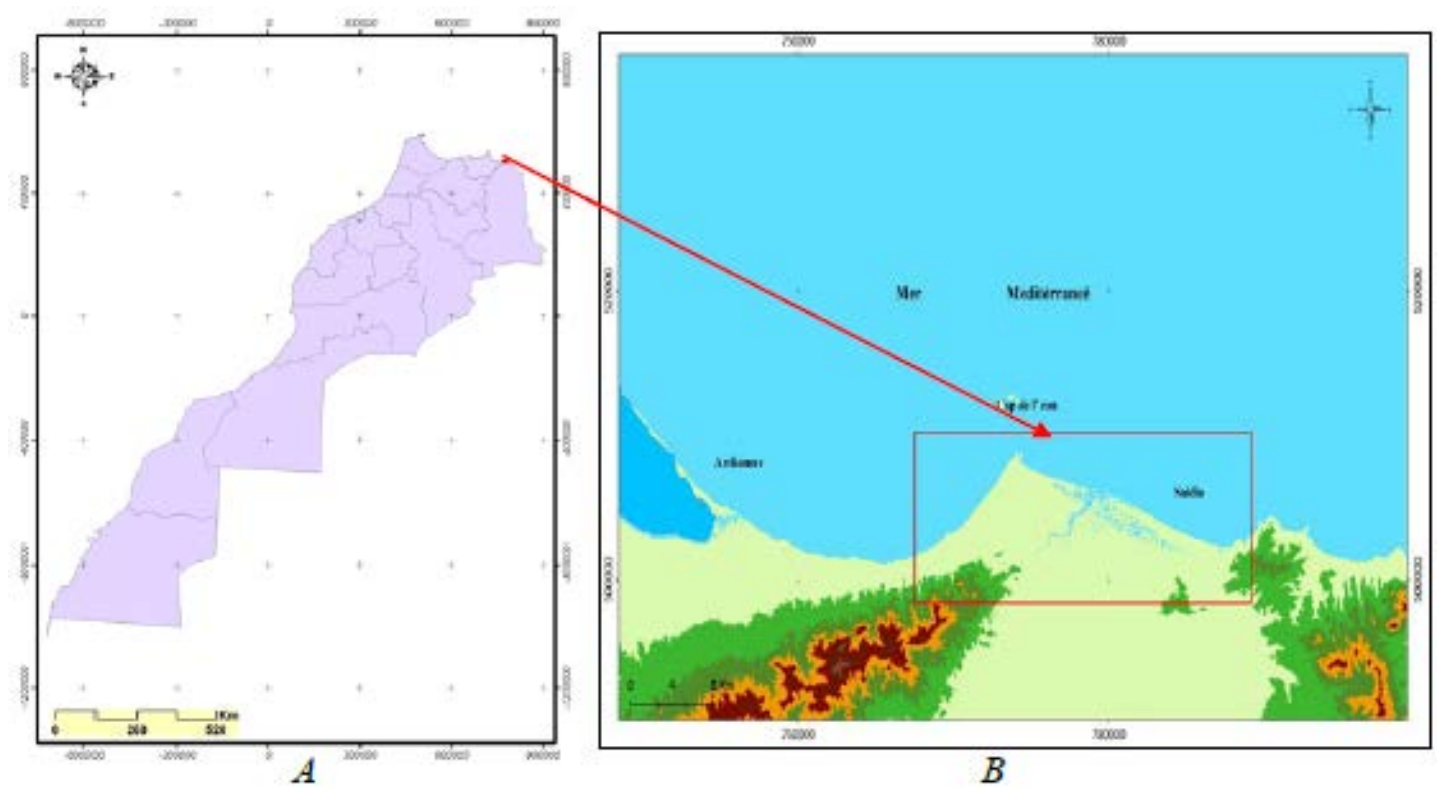

Figure 1. Localisation de la zone d'étude (B) par rapport au Maroc(A).

\section{Méthodes et données}

L'approche cartographique repose sur l'analyse multi date des photographies aériennes verticales en vue de suivre la mobilité de la ligne de rivage instantanée analysée par l'interprétation visuelle. La méthode s'appuie sur :

- le géo-référencement des photographies aériennes, suivant les repères des cartes topographiques à échelle 1:25000 et la projection Lambert Nord, Maroc, Zone 1. 


\section{XIII ${ }^{\text {èmes }}$ Journées Nationales Génie Côtier - Génie Civil \\ Dunkerque, 2-4 juillet 2014}

- l'assemblage des photographies disponibles (1958-1988, 2008).

- la superposition des différentes couches en utilisant un logiciel SIG (ArcGis 9.1). Les documents utilisés pour l'analyse diachronique sont reportés dans le tableau 1.

Pour l'analyse granulométrique, l'échantillonnage (30 échantillons superficiels) a été effectué le long de 10 profils perpendiculaires au rivage (estran, plage et dune) et réparti entre les sites de la côte sableuse et les sites de la côte rocheuse. Les pourcentages cumulés des différentes fractions granulométriques servent à établir les courbes granulométriques à l'aide d'un programme sous Excel. L'analyse de ces dernières se base sur les classifications proposées par FOLK et WARD (1957) et sont exprimées en unités phi (Ø).

Tableau.1. Base de données

\begin{tabular}{lll}
\hline Type de document & Date & Echelle \\
\hline Photo aérienne & 1958 & $1 / 20.000$ \\
Photo aérienne & 1988 & $1 / 20.000$ \\
Photo aérienne & 2008 & $1 / 20.000$ \\
\hline
\end{tabular}

\section{Etude des changements spatio-temporels du trait de côte entre 1958 et 2008}

Les informations tirées par de nombreuses études réalisées sur l'évolution du trait de côte du littoral à proximité de l'embouchure d'Oued Moulouya témoignent d'une part d'un recul entre 1959 et 2008 tout au long des secteurs qui voisinent l'embouchure d'Oued Moulouya et, d'autre part la régularisation du trait de côte de la rive gauche de l'embouchure de cet Oued (BOUMEAZA., 2002 ; BOUABDALLAH \& LARUE, 2009) La largeur du ce cours d'eau en contact avec la mer a considérablement diminué passant de $700 \mathrm{~m}$ en 1958 à $15 \mathrm{~m}$ seulement en 2008 conséquence de démantèlement de Delta (SBAI \& BOUAZZA, 2002). A l'échelle de Saïdia, le taux de retrait était de $68 \mathrm{~m}$, soit $1,12 \mathrm{~m} / \mathrm{an}$ (figure 2). L'application des logiciels de rectifications et de superposition sur des photographies aériennes espacées dans le temps nous a confirmé que la côte sableuse entre Cap de l'Eau et Oued Moulouya n'échappe pas à cette évolution à savoir que l'erreur a été estimée à \pm 1 mètre pour chaque photographie.

Le trait de côte en 1958: la photographique aérienne de 1958 présente des cheminements équilibrés des transits sédimentaires. Ainsi, l'avancement de la ligne du rivage à cette époque a été attaché largement à la régularité et l'abondance des apports fluviatiles qui ont contribué intensivement à l'édification d'un delta au front de la mer.

Ceux-ci reflètent la complémentarité du fonctionnement des éléments appartenant à deux genres d'hydrodynamique fluviatile et marine (la houle, la dérive littorale, les courants, le débit...).

Le trait de côte entre 1958 et 1988 : la régression des apports sédimentaires due aux aménagements fluviatiles édifiés successivement sur Oued Moulouya (barrages en construction en1958 et 1967), a inauguré une tendance à l'érosion qui a touché le profil 
longitudinal du trait de côte entre Cap de l'Eau et l'embouchure de la Moulouya. Dans ce sens, la photographie aérienne de 1988, enregistre un recul du trait de côte d'environ 200 m à l'échelle du delta de la rive gauche de l'embouchure de la Moulouya et d'environ $44 \mathrm{~m}$ au long des sites avoisinants cet embouchure.

Depuis 1981, date de l'installation du port de Cap de l'Eau, la ligne du rivage a connu un bouleversement spectaculaire avec une érosion attestée seulement dans les sites qui voisinent l'embouchure de la Moulouya. A l'inverse, le trait de côte proche du Cap de l'Eau a progressé d'environ $88 \mathrm{~m}$.

Le trait de côte entre 1988 et 2008 (figure $n^{\circ}$ 3) : Au cours cette période, deux types de tendance se distinguent1) une tendance à l'engraissement à proximité du port de Cap de l’Eau (+71 m), 2) une tendance à la l'érosion par recul du trait de côte dans les sites qui avoisinent l'embouchure de la Moulouya (-56 m).

Généralement, le trait de la côte a reculé de plus de $55 \mathrm{~m}$ par rapport à sa position en 1958, soit une moyenne de déplacement de 1,13 m/an.

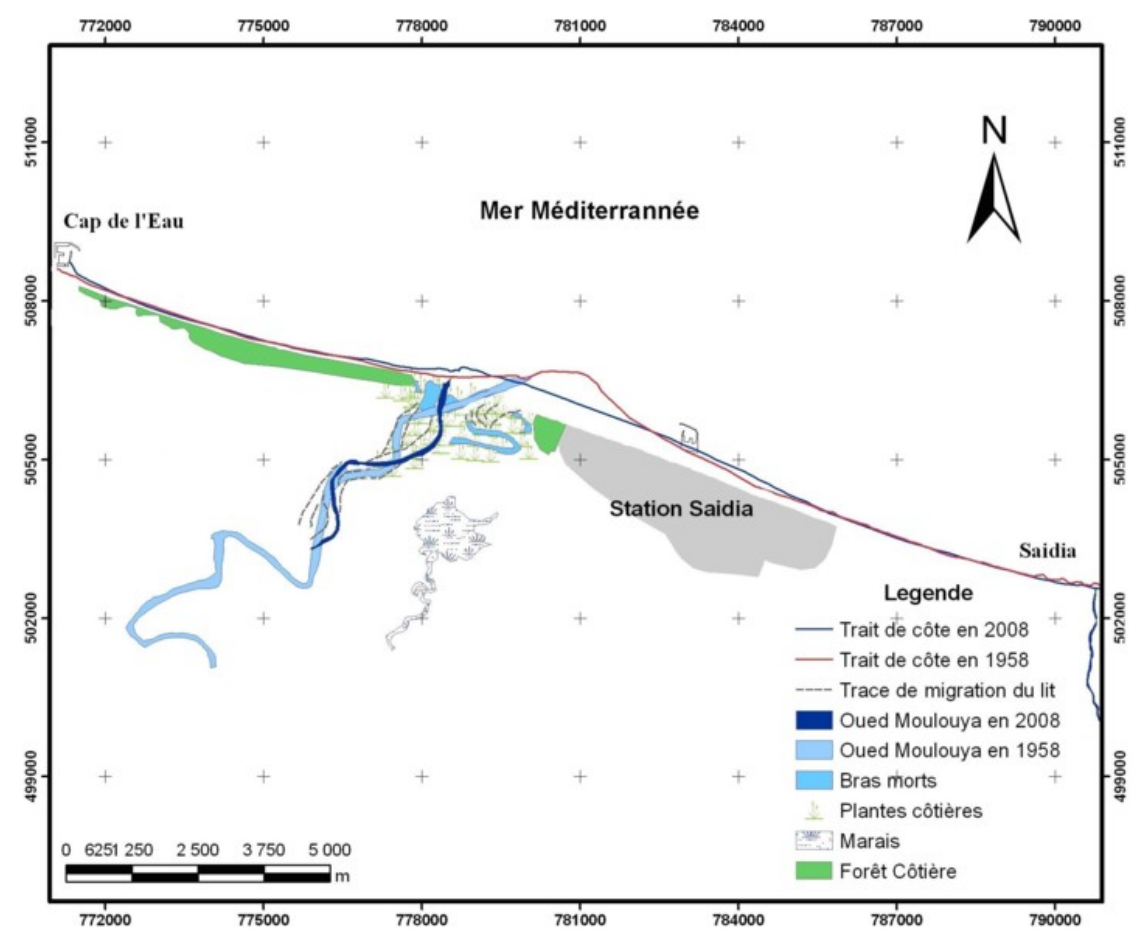

Figure 2. Evolution du trait de côte entre 1958 et 2008.

\section{Etude de la dynamique sédimentaire des compartiments de la plage}

Le littoral des Kebdana se compose de deux types de sable : les sables de la côte meuble à l'est du Cap de l'Eau, les sables de la côte rocheuse à l'ouest du Cap de l'Eau. Les classes granulométriques centrées entre $250 \mu \mathrm{m}$ et $125 \mu \mathrm{m}$ prédominent sur la côte sableuse. Cette fraction représente 94,7\% de sable de l'ensemble des échantillons. 


\section{XIII ${ }^{\text {èmes }}$ Journées Nationales Génie Côtier - Génie Civil \\ Dunkerque, 2-4 juillet 2014}

Les courbes cumulatives transversales dessinent un gradient granodécroissant de l'estran vers la dune pour la fraction des sables fins à très fins $(250 \mu \mathrm{m}-63 \mu \mathrm{m})$ à part les secteurs à proximité de la Moulouya, l'estran l'emporte sur les dunes.

Les courbes cumulatives longitudinales montrent que cette fraction appartenant à l'estran et à la plage s'affinent de l'embouchure vers le port à l'ouest (Site 6a, figure 4).

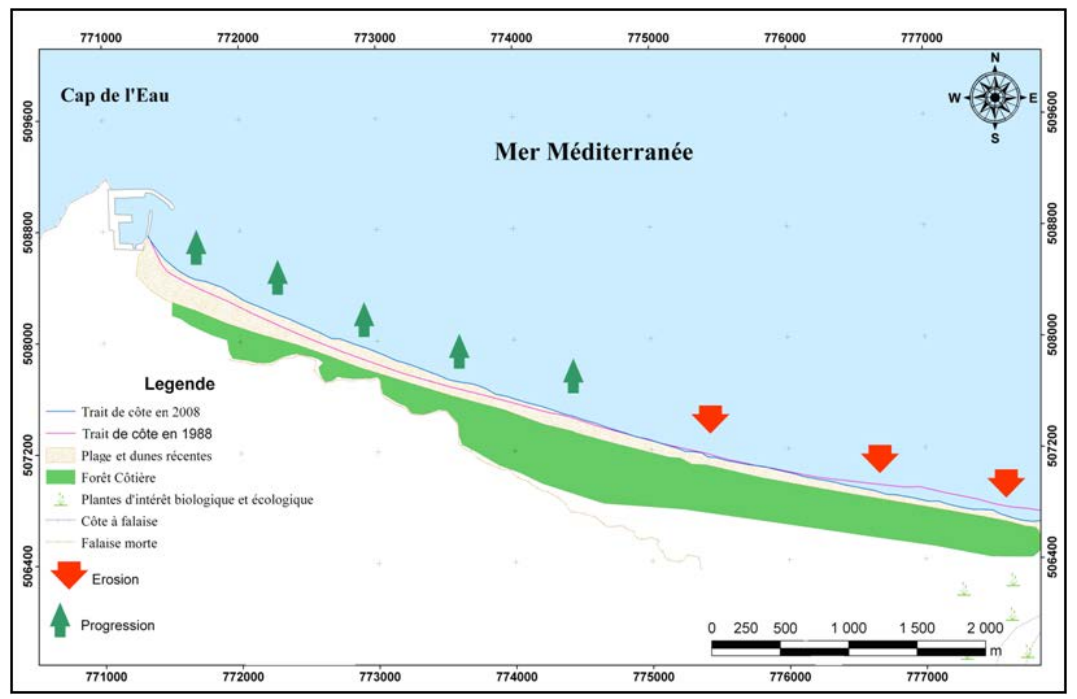

Figure 3. Evolution du trait de côte entre 1988 et 2008.

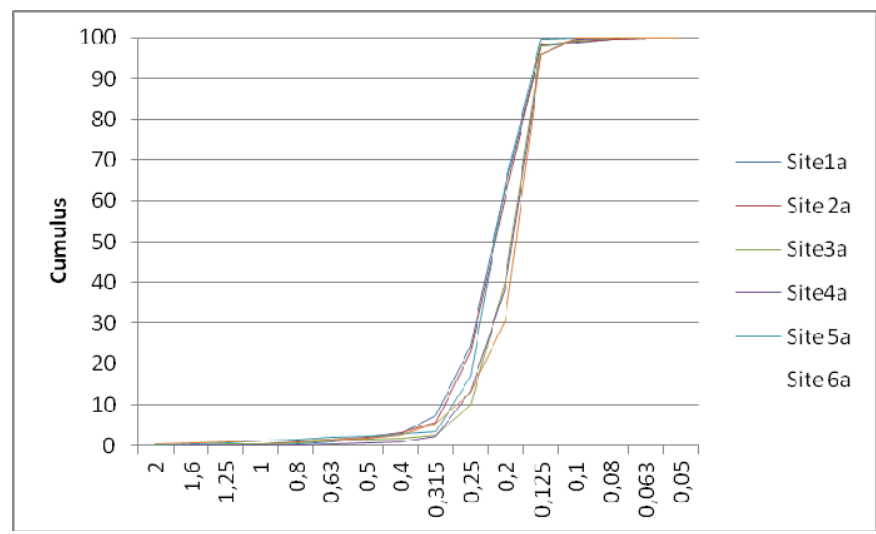

Figure 4. Courbes granulométriques de la fraction sableuses prélevée sur l'estran à l'embouchure de la Moulouya, Cap de l'Eau.

Les taux les plus élevés de la fraction à grain moyen se concentrent le long de l'estran à l'extrémité Est près de la Moulouya et au milieu de la plage.

Les compartiments de la côte sableuse montrent une homogénéité au niveau des paramètres granulométriques. Ces sédiments sont fins à moyens, avec une moyenne granulométrique entre 2, 65 et 2,31. Ils sont bien classés, et montrent une asymétrie vers les petites tailles. L'enrichissement de l'estran et des dunes en sables fins est due à la dynamique du transport marin et éolien. La dynamique du transport marin effectuée par 
les petites marées assure l'évacuation des particules fines d'origine marine et fluviatile vers l'estran. La déflation et le transport par les vents contribuent à accroitre le stock dunaire à partir des grains de petite taille disponibles. Cette constatation est confirmée par la prédominance de la proportion des grains émoussés-luisants sur celle des grains émoussés-non usés.

Concernant la côte rocheuse à l'ouest de Cap de l'Eau, la répartition granulométrique révèle une hétérogénéité du matériel marquée par un mélange de sables moyens à grossiers à très grossiers : entre $250 \mu \mathrm{m}$ et $2 \mathrm{~mm}$ avec un écart à l'échelle des valeurs des moyennes granulométrique (0,45 et 1,59 , figure 5$)$.

Les valeurs de l'écart type sont très variables $(0,30$ - 1,16) indiquent des sables bien classés à très mal classés. Ainsi, cette hétérogénéité atteste de la confluence entre les apports des oueds lors des crues et l'intensité de l'érosion et la mobilisation des sédiments issues des falaises vives adjacentes à travers une action hydrodynamique marine relativement forte et variable en intensité.

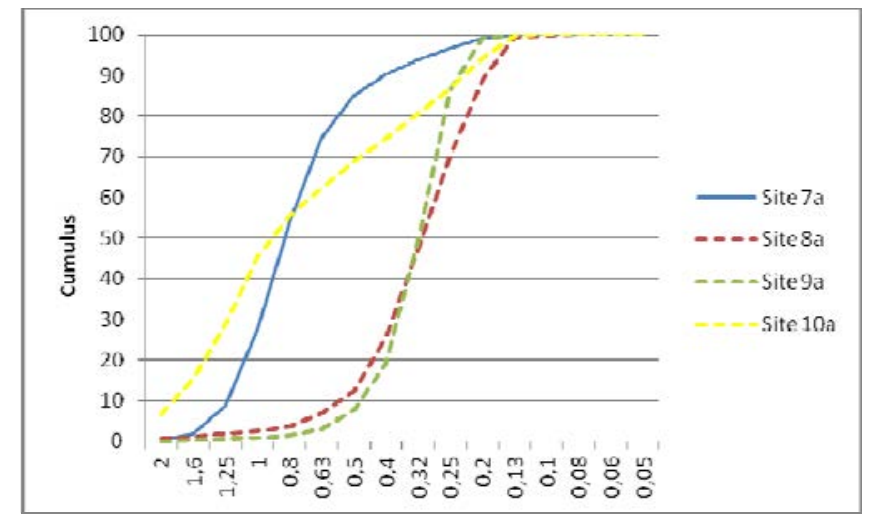

Figure 5. Courbes granulométriques de la fraction sableuses prélevée sur l'estran à l'ouest de Cap de l'Eau.

\section{Impact des projets d'aménagements et les changements climatiques sur la dynamique côtière}

\subsection{Erosion de la plage : impact des barrages et effet du changement climatique}

La ligne de rivage à proximité de l'embouchure de la Moulouya a reculé et s'est accompagnée d’une diminution de la largeur des plages. PASKOFF (1994) met en relation l'amaigrissement et la disparition des plages avec le processus d'élévation du niveau de la mer. Corrélativement à cette élévation les vagues qui déferlent plus près de la ligne de rivage, libèrent leur énergie plus haut sur l'estran et modifient le profil des plages surtout si de nouveaux apports bloqués en rivière par les barrages ne viennent compenser les pertes. L'élévation probable du niveau de la mer de $2 \mathrm{~m}$ durant les prochaines décennies risquent d'inonder la côte sableuse, les plaines côtières, la 


\section{XIII ${ }^{\text {èmes }}$ Journées Nationales Génie Côtier - Génie Civil \\ Dunkerque, 2-4 juillet 2014}

nouvelle station balnéaire de Saïdia (figure 6). Quant au scénario $>2 \mathrm{~m}$, l'arrière-pays est directement ciblé d'une éventuelle submersion.

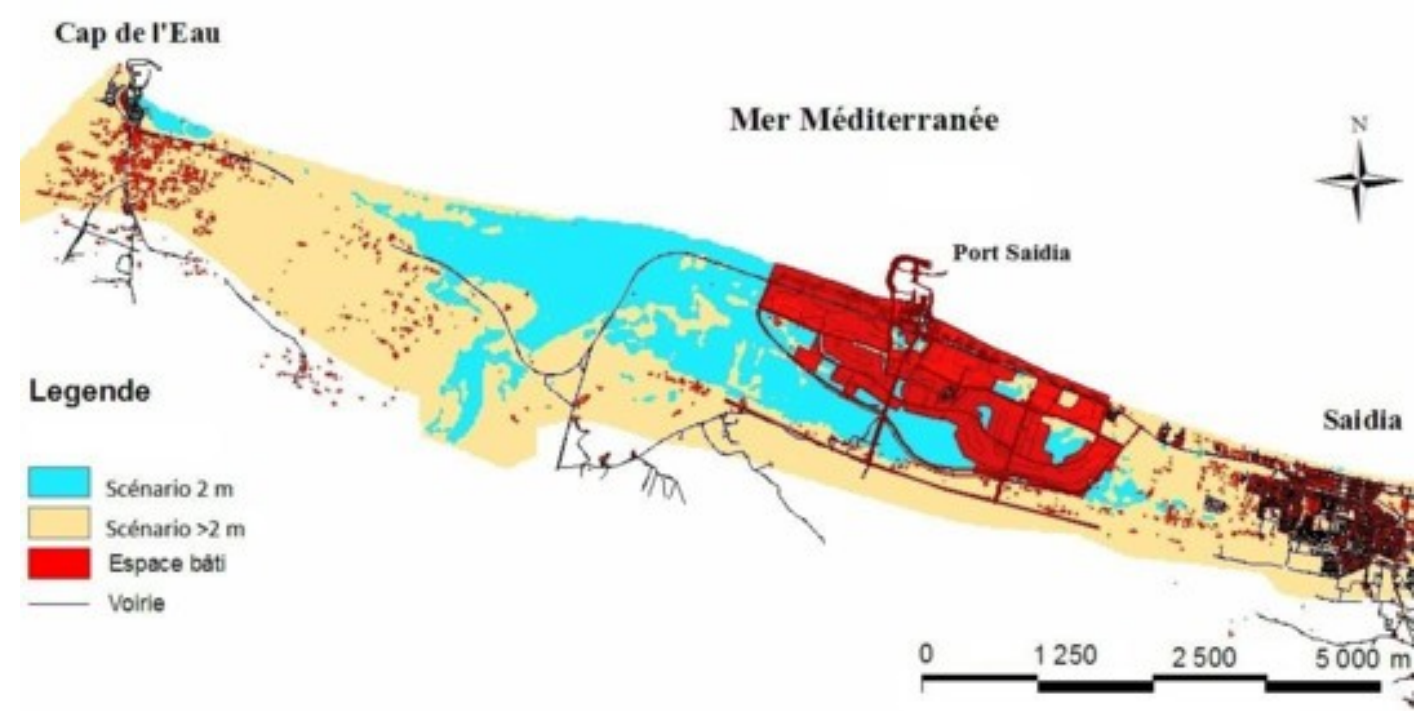

Figure 6. Zones à risque d'inondation en cas d'élévation du niveau de la mer de $2 \mathrm{~m}$ et de plus de 2 m. Source : Model numérique du terrain.

6.2 Ensablement de la plage et genèse des avants côtes à Cap de l'Eau : effet du port

L'accumulation du sable se développe dans le secteur ouest de la plage derrière la jetée du port secondaire qui s’implante perpendiculairement à la ligne de la côte. Cette jetée mal localisée freine les sédiments et amène une déviation au courant latéral qui entraîne une perturbation au transit latéral affectant les échanges longitudinaux et transversaux entre les secteurs de la plage. Ainsi, la forme du profil longitudinal du trait de côte de ces sites apparait avoir une concavité tournée vers le nord-ouest ou sous forme d'un triangle dont l'un de ses côtés prograde progressivement vers la mer. On note également la création d'une nouvelle génération des barres d'avant plage. L'apparition de ces barres qui sont riches en matériaux est due à l'effet du port qui a déplacé la zone de déferlement vers le large lors des hautes marées. Ceci s'ajoute à l'action des houles longues perpendiculaires à la côte en provenance du secteur NE. Les caractères de ces houles prédominantes dans ces sites favorisent le transport des sédiments vers le large et la construction de barres d'avant côte.

Ces barres de sable, d’une longueur totale de 0,5 km viennent se coller contre la jetée barrant ainsi l'entrée du port. Une bonne partie de ces sables pénètre ce dernier en y provoquant son ensablement, ce qui nécessite constamment des opérations de dragage pour assurer l'accès au port. Les mesures faites par les services concernées montrent un taux d'ensablement très élevé pendant la période allant de 1991 à 2013. La quantité des sédiments dragués a dépassé $158000 \mathrm{~m}^{3}$ en 1996 et $65000 \mathrm{~m}^{3}$ en 2013. Les sables sont remis en mer dans un lieu très proche de port de Cap vers le Nord. Ce lieu n'est pas 
adéquat pour compenser des mouvements de dégraissement de plage parce que son choix n’a été pas basé sur une étude préalable de l’hydrodynamique côtière.

6.3 L'impact de la bétonisation de la dune bordière sur l'équilibre sédimentaire

Les constructions édifiées sur la haute plage (corniche, club) empêchent la mer de prélever sur ces milieux des matériaux susceptibles d'alimenter la basse plage lors des tempêtes. Ainsi, l'action érosive de la houle et des courants côtiers se concentre alors sur la plage elle-même en transformant la composition granulométrique par prélèvements des sables fins et concentration des sables grossiers. En outre la destruction des dunes au profil des projets immobilières ne fait que disparaitre la réserve des sédiments nécessaire à l'équilibre des côtes d’après (PASKOFF, 1996).

\section{Conclusion et recommandation}

Le littoral entre le Cap de l’Eau et Saïdia est en état de déséquilibre. Les éléments qui contrôlent le bon fonctionnement de la régularisation du trait de côte subissent jusqu’à l'instant des effets indésirables dus aux aménagements fluviatiles, portuaires, touristiques. Ainsi le processus de l'anthropisation et la littoralisation des côtes dans l'aire d'étude s'intègre dans le cadre de cette réalité mondiale. La préservation de ce patrimoine environnemental fragile et paysager encore riche nécessite une gestion intégrée. Il est proposé pour en valoriser les potentialités dans le futur de proumouvoir une méthode de gestion basée sur une approche participative.

\section{Références bibliographiques}

BOUABDALLAH M., LARUE J-P. (2009). Évolution du littoral de la baie de Saïdia : dynamique naturelle et impacts des aménagements (Maroc oriental). Physio-Géo, Vol. 3, pp 113-130.

BOUMEAZA T. (2002). Le cours inférieur de la Moulouya : morphologie quaternaire, dynamique fluviale et morphologie côtière du littoral entre Kebdana et Saïdia (Maroc oriental, Maroc). Thèse de doctorat, Univ. de Liège, Fac. Sc., 340 p.

FOLK R.L., WARD W.C. (1957). Brazos river bar: A study in the signification of grainsize parameters. Journal of Sedimentary Petrology, Vol. 27; pp 3-26. http://dx.doi.org/10.1306/74D70646-2B21-11D7-8648000102C1865D

PASKOFF R. (1994). Les littoraux, impact des aménagements sur leur évolution. Edit Masson géographie, Paris, $2^{\mathrm{e}}$ édition, $256 \mathrm{p}$.

PASKOFF R. (1996). Aménagement du littoral et protection de l'environnement en France. Armand Colli, l'information géographique, Vol. 60, pp 157-166.

SBAI A., BOUAZZA A. (2002). Evolution géomorphologique et dynamique récente de la plaine littorale de Saïdia (Maroc NORD-EST). In: Le littoral méditerranéen oriental : Diagnostic et choix de développement et d'aménagement. Colloque national, 13-14 février 2002, Oujda, pp 3-30. 